\title{
Majorana oscillations and parity crossings in semiconductor nanowire-based transmon qubits
}

\author{
J. Ávila $\odot,{ }^{1}$ E. Prada $\odot,{ }^{2}$ P. San-Jose $\odot,{ }^{1}$ and R. Aguado $\odot^{1}$ \\ ${ }^{1}$ Instituto de Ciencia de Materiales de Madrid (ICMM), Consejo Superior de Investigaciones Científicas (CSIC), \\ and Research Platform on Quantum Technologies (CSIC), Sor Juana Inés de la Cruz 3, 28049 Madrid, Spain \\ ${ }^{2}$ Departamento de Física de la Materia Condensada, Condensed Matter Physics Center (IFIMAC) and Instituto Nicolás Cabrera, \\ Universidad Autónoma de Madrid, E-28049 Madrid, Spain
}

(Received 28 February 2020; revised 17 June 2020; accepted 20 August 2020; published 25 September 2020)

\begin{abstract}
We show that the microwave (MW) spectra in semiconductor-nanowire-based transmon qubits provide a strong signature of the presence of Majorana bound states in the junction. This occurs as an external magnetic field tunes the wire into the topological regime and the energy splitting of the emergent Majorana modes oscillates around zero energy owing to their wave function spatial overlap in finite-length wires. In particular, we discuss how these Majorana oscillations, and the concomitant fermion parity switches in the ground state of the junction, result in distinct spectroscopic features-in the form of an intermittent visibility of absorption lines-that strongly deviate from standard transmon behavior. In contrast, nonoscillating zero modes, such as topologically trivial Andreev bound states resulting from sufficiently smooth potentials, exhibit an overall standard transmon response. These differences in the MW response could help determine whether the junction contains topological Majoranas or not.
\end{abstract}

DOI: 10.1103/PhysRevResearch.2.033493

\section{INTRODUCTION}

Superconducting islands based on Josephson junctions (JJs) shunted by a capacitor are the key element in qubits based on superconducting circuits [1-3]. Their physics is controlled by the ratio $E_{J} / E_{C}$ between the Josephson coupling $E_{J}$ and the charging energy $E_{C}$. This interplay is described by the Hamiltonian $H=4 E_{C}\left(\hat{N}-n_{g}\right)^{2}+V_{J}(\hat{\varphi})$, where $V_{J}(\hat{\varphi})=$ $-E_{J} \cos \hat{\varphi}$ [4]. Here $\hat{N}$ is the Cooper-pair number, conjugate to the superconducting phase $\hat{\varphi}$, and $n_{g}=Q_{g} / 2 e$ is a gate-induced offset. Recent experimental efforts are pushing the standard operation limits in order to have JJs compatible with electrical gating and high magnetic fields. This compatibility is a crucial step to reach a regime relevant for microwave (MW) readout of topological qubits based on Majorana bound states (MBSs) [5-16]. Various options include semiconductors [17-23] and van der Waals heterostructures [24-26].

We here focus on JJs based on semiconducting nanowires (NWs). In the presence of an external Zeeman field $B$, the NWs can be driven into a topological superconductor phase [27-30] where MBSs couple across the JJ with strength $E_{M}$ and coherently interact with the superconducting island degrees of freedom. Our fully microscopic description allows one to study the detailed magnetic field evolution of the NW junction, including the emergence of MBSs in the

Published by the American Physical Society under the terms of the Creative Commons Attribution 4.0 International license. Further distribution of this work must maintain attribution to the author(s) and the published article's title, journal citation, and DOI. topological phase, and to address the possibility of nontopological robust zero modes that appear in junctions with spatially smooth potentials [31-35]. Since both $E_{J}$ and $E_{M}$ depend on $B$, this allows one to unveil new physics from the $E_{J} / E_{C} \lesssim 1$ Cooper-pair box (CPB) to the $E_{J} \gg E_{C}$ transmon regimes depending on the ratio $E_{M} / E_{C}$. The hitherto unexplored $E_{M} / E_{C} \gg 1$ regime presents parity-dependent $\mathrm{MW}$ signatures that map parity switches in the NW spectrum owing to the oscillatory energy splitting of overlapping Majoranas. In contrast, nontopological modes pinned to zero energy yield a standard transmon spectrum. Hence, MW spectroscopy provides a powerful tool to distinguish between both scenarios.

\section{MODEL}

The Josephson potential is defined, on a microscopic level, as the operator $V_{J}(\varphi)=\frac{1}{2} \check{\boldsymbol{c}}^{\dagger} H_{\mathrm{BdG}}(\varphi) \check{\boldsymbol{c}}$, where $\check{\boldsymbol{c}}=$ $\left(c_{i \uparrow}, c_{i \downarrow}, c_{i \uparrow}^{\dagger}, c_{i \downarrow}^{\dagger}\right)$ are Nambu spinors and $H_{\mathrm{BdG}}$ is the Bogoliubov-de Gennes (BdG) Hamiltonian

$$
H_{\mathrm{BdG}}(\varphi)=\left(\begin{array}{cc}
H_{\mathrm{NW}} & \Delta(x, \varphi) \\
\Delta(x, \varphi)^{\dagger} & -H_{\mathrm{NW}}^{*}
\end{array}\right) .
$$

$H_{\mathrm{NW}}$ consists of two (left/right) segments of length $L_{S}$ with normal Hamiltonians $H_{L / R}$, coupled across a short weak link of transparency $T_{N} \in[0,1]$. Each NW segment contains all the microscopic details (Rashba coupling $\alpha$, Zeeman field $B$, and chemical potential $\mu$ ) and is described by a single-band model $H_{L / R}=\frac{p_{x}^{2}}{2 m}-\mu-\frac{\alpha}{\hbar} \sigma_{y} p_{x}+B \sigma_{x}$ (with $p_{x}=-i \hbar \partial_{x}$ the momentum operator and $\sigma_{i}$ Pauli matrices in spin space). These NWs undergo a topological phase transition at $B_{c} \equiv$ $\sqrt{\Delta^{2}+\mu^{2}}$ with the appearance of MBSs at their edges [see 

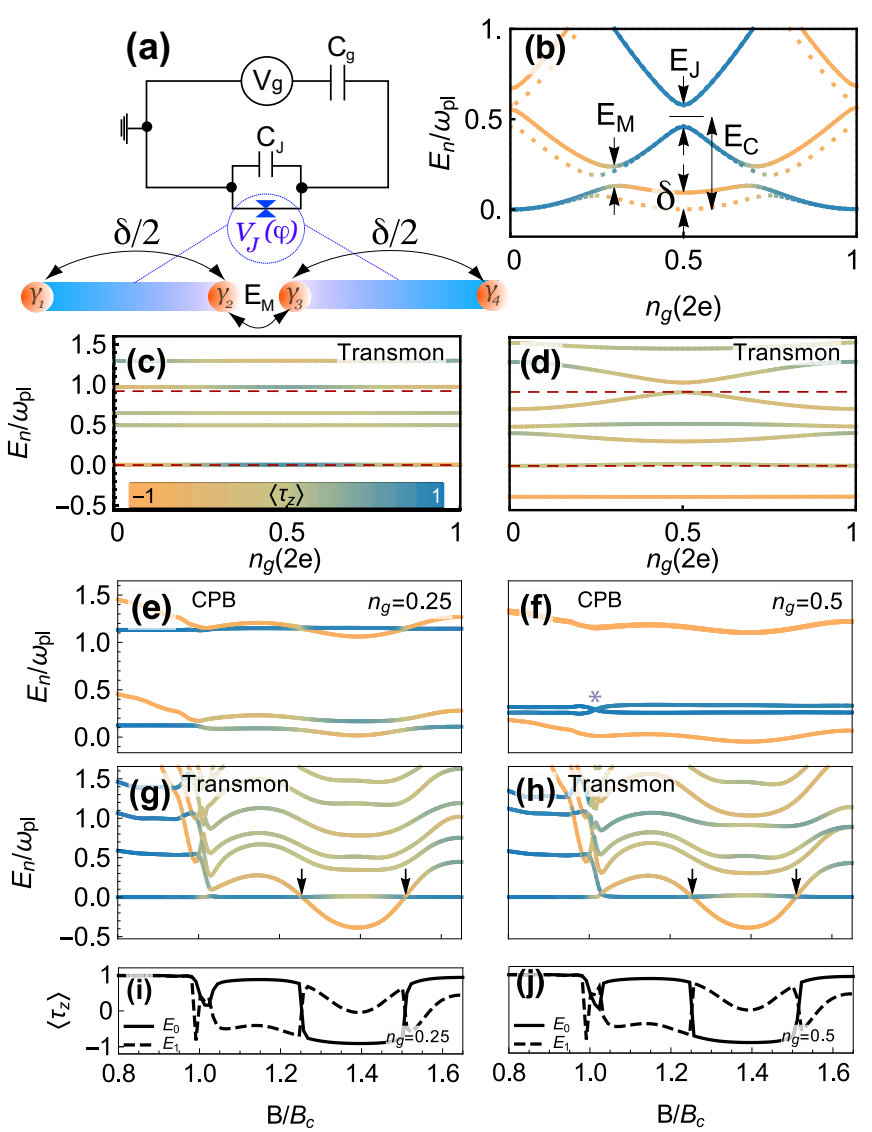

FIG. 1. Sketch and spectrum of a NW-based superconducting island. (a) Simplified transmon/CPB circuit. $E_{J}$ is implicit in the Josephson potential $V_{J}(\varphi)$, while the combination of a shunting capacitor $C_{J}$ and the gate capacitance $C_{g}$ define the charging energy $E_{C}=e^{2} / 2\left(C_{J}+C_{g}\right)$. The presence of MBSs $\gamma_{i}$ gives rise to two new energy scales: the Majorana splitting $\delta / 2$ and the Majorana coupling $E_{M}$. (b) Spectrum of the island versus $n_{g}=V_{g} /\left(2 e C_{g}\right)$ in the CPB regime $\left(E_{J} / E_{C}=0.5, E_{M} / E_{C} \sim 0.12\right)$ and for $B \sim B_{c}$. Blue/orange colors denote fermionic even/odd parities, while intermediate gradient signals parity mixing. The dotted lines show a slightly different $B$ field with $\delta=0$. (c) Same as (b) but in a transmon regime with $E_{J} / E_{C}=25, E_{M} / E_{C} \sim 6.5$ (dashed lines show the original transmon lines at $B=0$ ). Panel (d) shows the same transmon case but for $\delta \neq 0$ $\left(B=1.4 B_{c}\right)$. (e), (f) Magnetic field dependence for the CPB in (b), at $n_{g}=0.25$ and $n_{g}=0.5$, respectively. In this latter case, the topological transition (where $E_{J} \rightarrow 0$ ) can be seen as a closing of the qubit frequency (asterisk). (g), (h) Same as (e), (f) but for the transmon (arrows mark parity crossings). (i), (j) Parity polarization $\left\langle\hat{\tau}_{z}\right\rangle$ of the two lowest states in (g), (h). At parity crossings, the polarization jumps. Rest of parameters: $L_{S}=2.2 \mu \mathrm{m}, \tau=0.8, \mu=0.5 \mathrm{meV}$, $\Delta=0.25 \mathrm{meV}$.

blowup in Fig. 1(a)]. $\Delta(x, \varphi)=i \sigma_{y} \Delta e^{ \pm i \varphi / 2}$ (where the \pm corresponds to $x \in L / R$, respectively) is the induced pairing term [36,37]. While $V_{J}(\varphi) \sim-E_{J} \cos (\varphi)$ is a good approximation at $B=0$ in the $T_{N} \rightarrow 0$ tunneling limit, it can strongly deviate from this form under relevant experimental conditions $[1,19,37]$.

Starting from Eq. (1), our goal is to derive a quantitatively precise low-energy approximation for $V_{J}$ that retains both standard Josephson events due to Cooper-pair tunneling, as well as anomalous Majorana-mediated events where a single electron is transferred across the junction. While the total fermion parity $n \bmod 2$ is conserved (where $n=n_{L}+n_{R}$ and $n_{L, R}$ are the fermion occupations in the left/right segments of the junction), an anomalous tunneling term changes the parity $n_{L / R} \bmod 2$ on each superconducting left/right segment. For instance, assuming even global parity, Majoranamediated tunneling corresponds to the process $|0\rangle \equiv \mid n_{L}=$ $\left.0, n_{R}=0\right\rangle \Longleftrightarrow|1\rangle \equiv\left|n_{L}=1, n_{R}=1\right\rangle$. (In what follows, even/odd parity will always refer to the partial fermion parity $n_{L} \bmod 2=n_{R} \bmod 2$.) Physically, this suggests that to compute an effective low-energy $V_{J}(\varphi)$, it is convenient to distinguish two contributions, $V_{J}(\varphi)=V_{J}^{\text {bulk }}(\varphi)+H_{\mathrm{BdG}}^{\text {sub }}$. The first one takes into account the bulk contribution of BdG levels above the gap to the ground state (GS) energy $V_{J}^{\text {bulk }}(\varphi)=-\sum_{\epsilon_{p}>\Delta} \epsilon_{p}(\varphi)$. The second contribution corresponds to the subgap sector (four subgap states resulting from two electron-hole copies of two spin-resolved subgap states), which can be described in terms of four Majorana operators, $\gamma_{1,2} \in L$ and $\gamma_{3,4} \in R$, interacting pairwise [38,39]:

$$
\begin{aligned}
H_{\mathrm{BdG}}^{\mathrm{sub}}(\varphi)= & i \lambda_{12} \gamma_{1} \gamma_{2}+i \lambda_{13}(\varphi) \gamma_{1} \gamma_{3}+i \lambda_{14}(\varphi) \gamma_{1} \gamma_{4} \\
& +i \lambda_{23}(\varphi) \gamma_{2} \gamma_{3}+i \lambda_{24}(\varphi) \gamma_{2} \gamma_{4}+i \lambda_{34} \gamma_{3} \gamma_{4}
\end{aligned}
$$

\section{PROJECTION METHOD}

To derive the different $\lambda_{i j}$ from microscopics, we first calculate the Nambu spinors $\breve{\psi}_{i s}^{0}$ of the full/empty $(i= \pm)$ lowest subgap eigenstate of each decoupled $s=L, R$ segments. These states span a fermion basis $\left\{c_{L}, c_{L}^{\dagger}, c_{R}, c_{R}^{\dagger}\right\}$ of our four-dimensional projection space, in terms of which we can write fermion numbers as $n_{L}=c_{L}^{\dagger} c_{L}=\left(1+i \gamma_{1} \gamma_{2}\right) / 2$ and $n_{R}=c_{R}^{\dagger} c_{R}=\left(1+i \gamma_{3} \gamma_{4}\right) / 2$. Performing the low-energy projection

$$
\left(\mathcal{H}^{-1}\right)_{i^{\prime} s^{\prime}, i s}=\left\langle\psi_{i^{\prime} s^{\prime}}^{0}|G(\omega=0)| \psi_{i s}^{0}\right\rangle,
$$

where $G(\omega)=\left(\omega+i \varepsilon-H_{\mathrm{BdG}}\right)^{-1}$ is the resolvent of the full $\mathrm{BdG}$ Hamiltonian, Eq. (2) can be written as $H_{\mathrm{BdG}}^{\mathrm{sub}}=$ $\frac{1}{2} \breve{\psi}^{0 \dagger} \mathcal{H} \breve{\psi}^{0}$, which yields the different $\lambda_{i j}$. After projecting onto the parity basis $\{|p\rangle\}=\{|0\rangle,|1\rangle\}$, the final effective Hamiltonian reads

$$
H=\left[4 E_{C}\left(-i \partial_{\varphi}-n_{g}\right)^{2}+V_{J}^{\text {bulk }}(\varphi)\right] \mathbb{1}+V_{J}^{\text {sub }}(\varphi) .
$$

It describes two different parity copies of a superconducting island, which are mixed through a nondiagonal term $V_{J p^{\prime} p}^{\text {sub }}(\varphi)=\left\langle p^{\prime}\left|H_{\mathrm{BdG}}^{\text {sub }}\right| p\right\rangle$. The parity content of the eigenstates of Eq. (4) is calculated by a projection onto the parity axis defined by $\hat{\tau}_{z} \equiv|0\rangle\langle 0|-| 1\rangle\langle 1|$.

Since Eq. (4) is derived by projecting $H_{\mathrm{BdG}}$, the resulting coefficients in Eq. (2) depend on all the microscopic details of the problem, $\lambda_{i j}\left(B, L_{S}, \mu, \alpha\right)$, instead of just being constant parameters like in previous effective models $[9,10]$. Thus, $H$ is able to describe, in particular, the full magnetic field evolution of the junction (from trivial to topological), which is governed by three relevant $B$-field-dependent microscopic energy scales [37]: the Josephson coupling $E_{J}=$ $\int_{0}^{2 \pi} \frac{d \varphi}{\pi}\left[V_{J}^{\text {bulk }}(\varphi)+V_{J 00}^{\text {sub }}(\varphi)\right] \cos (\varphi)$, the energy splitting between different fermionic parities $\delta=V_{J 11}^{\mathrm{sub}}(\varphi)-\left.V_{J 00}^{\mathrm{sub}}(\varphi)\right|_{\varphi=0}$ 
and the Majorana coupling $E_{M}=\int_{0}^{2 \pi} \frac{d \varphi}{\pi} V_{J 01}^{\mathrm{sub}}(\varphi) \cos (\varphi)$. Moreover, we can also analyze other possibilities, including the emergence of robust zero modes at $B \ll B_{c}$ (without a topological bulk) in wires with spatially inhomogeneous $\mu(x)$ [40].

\section{RESULTS}

In what follows, the ratio $E_{J} / E_{C}$ and the plasma frequency $\omega_{p l} \equiv \sqrt{8 E_{J} E_{C}} / \hbar$ are defined with respect to the zero-field junction, for which a microscopic calculation of $I_{c}[36,41,42]$ gives $E_{J} \equiv \hbar I_{c}(B=0) / 2 e$ at fixed $E_{C}$. In Fig. 1 the calculated fermionic parity content is represented by different colors (even/odd=blue/orange for $\left\langle\hat{\tau}_{z}\right\rangle= \pm 1$ ). In the CPB limit each even (odd) parabola in the spectrum [see Fig. 1(b)] has a minimum at $n_{g}=m+n_{g}^{0}$, where $m \in \mathbb{Z}$ and $n_{g}^{0}=0(0.5)$ (only the $m=0,1$ cases are shown). Odd parabolas are energy shifted from even ones by an amount $\delta$. For $B=0$ (not shown), the spectrum is $2 e$ periodic and the energy shift is exactly $\delta=2 \Delta$, since each extra fermion must overcome an energy gap $\Delta$ on each NW segment. Increasing $B$, the gap gets reduced until an odd GS around $n_{g}=0.5$ is possible when $\delta<E_{C}$. In the $\delta \rightarrow 0$ limit (dotted curve), both parity sectors have minima at zero energy and the periodicity becomes $e$ $[43,44]$. Furthermore, MBSs coherently mix parities around $n_{g}=0.25$ and $n_{g}=0.75[9,10,16]$. In the transmon limit with $\delta=0$, Fig. 1(c), strong parity mixing occurs for all $n_{g}$, which manifests as splitting of the lines with almost zero parity polarization $\left\langle\hat{\tau}_{z}\right\rangle \approx 0$. At a different $B$ corresponding to $\delta \neq 0$ [Fig. 1(d)], the transmon spectrum shows charge dispersion and an overall $2 e$ periodicity. This result illustrates how nominally identical transmons depend on microscopic properties of the NWs [in this case, the ratio $\xi_{M} / L_{S}$, with $\xi_{M}$ the Majorana coherence length [45], which governs $\delta(B)$ [46]]. Moreover, the finite $\delta \neq 0$ polarizes the GS parity (now of odd character for all $n_{g}$ ).

Next, we focus on the magnetic field evolution of the island spectrum. In the CPB regime, this evolution strongly depends on gate (owing to the large charge dispersion). Since parity mixing occurs near $n_{g}=0.25$, the $B$-field dependence at this gate considerably differs from the one at $n_{g}=0.5$, where parity mixing is negligible [compare Figs. 1(e) and 1(f)]. On the other hand, in the transmon limit the spectrum mimics Majorana oscillations. After each parity crossing [see arrows in Figs. 1(g) and 1(h)], the parity polarization $\tau_{z}$ jumps [Figs. 1(i) and 1(j)], which reflects changes in the parity character of the GS (change of color from blue to orange and back). This behavior occurs for all $n_{g}$. Switches of GS parity are possible since, for the single-band case considered here, $E_{M} \sim E_{J}[36,37,41,42,47]$. Consequently, in a transmon regime with $E_{J} / E_{C} \gg 1$, the ratio $E_{M} / E_{C}$ is not small [48]. This relevant regime has hitherto remained unexplored, even at the level of the effective models in Refs. $[9,10,16]$, which focus on the opposite $E_{M} / E_{C} \ll 1$ regime where the large charging energy prevents changes in the GS parity [37]. This would require much larger $E_{C}$ (which is detrimental since it induces charge dispersion) or very small Majorana couplings $E_{M} \ll E_{J}$. The latter can be, however, somewhat difficult to reach in a few-channel topological wire: while more than one channel, but not many, can contribute to $E_{J}[19,49,50]$, the
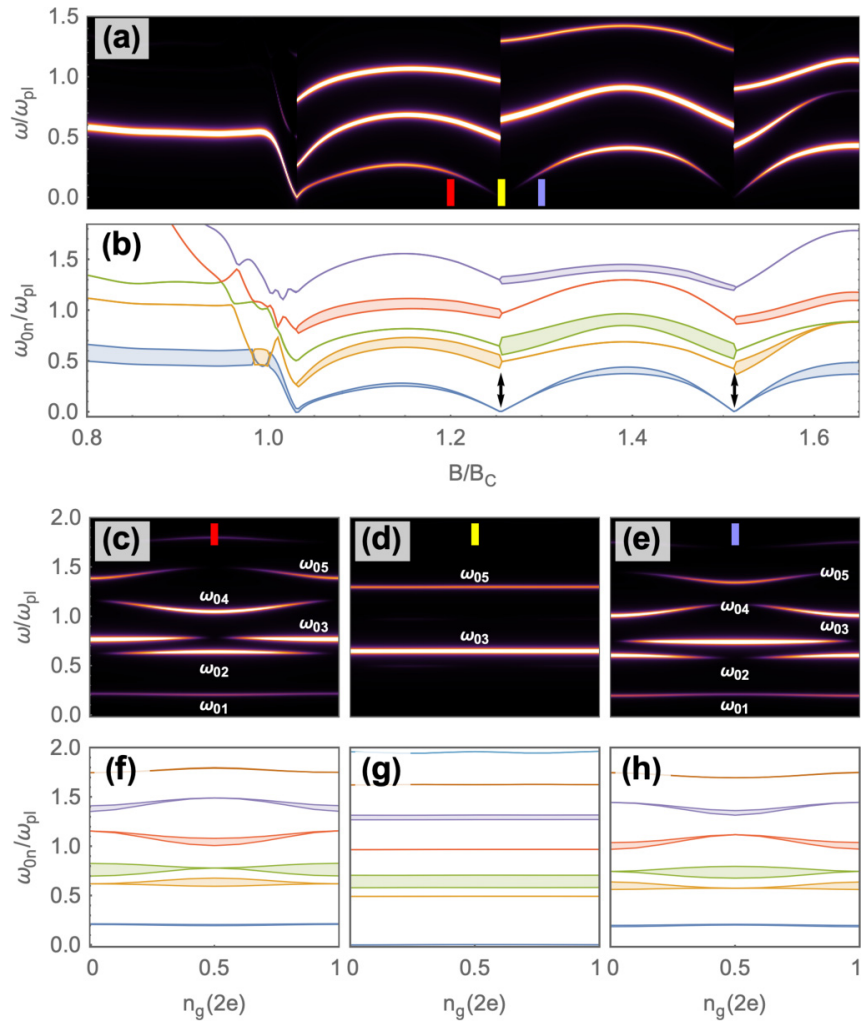

FIG. 2. MW spectroscopy of a NW-based transmon. (a) Contour plot of MW absorption spectrum $S_{N}(\omega)$ versus $\omega$ and $B / B_{c}$ at $n_{g}=0.5$. Bright lines signal allowed transitions in the MW response. Spectral holes in the $n=1$ transition line and abrupt jumps in the $n>1$ ones, where lines suddenly disappear/appear, coincide with parity crossings in the GS owing to Majorana oscillations. (b) Transition frequencies and spectral weights (shadowed widths). At minima of the oscillations (arrows), the spectral weight of different transitions gets exchanged. (c)-(h) $n_{g}$ dependence. Same parameters as the transmon in Fig. 1.

value of $E_{M}$ is of the order of the topological minigap [37]. These parity switches in the GS have important consequences for MW spectroscopy, as we discuss next.

\section{MW SPECTROSCOPY}

The linear-response MW spectrum can be written as $S_{N}(\omega)=\sum_{n}|\langle n|\hat{N}| 0\rangle|^{2} \delta\left(\omega-\omega_{n 0}\right)$, where $\Psi_{n}$ are the eigenstates of Eq. (4) and $\omega_{0 n}=\left(E_{n}-E_{0}\right) / \hbar$. In what follows we focus on the transmon limit with $E_{M} / E_{C} \gtrsim 1$. A full discussion about other relevant regimes, including the CPB and the transmon regime with $E_{M} / E_{C} \ll 1$, can be found in Ref. [37]. In Fig. 2(a) we plot the magnetic field dependence of the MW spectrum of a transmon. Majorana oscillations and parity switches in Figs. 1(h) and 1(j) are found to result in abrupt spectroscopic changes at $B$ fields where Majorana oscillations have nodes. They include spectral holes in the first transition $\omega_{01}$ (a transition within the ground state manifold which is visible thanks to the Majorana-induced parity mixing) accompanied by higher transition lines suddenly disappearing/appearing. The spectral holes can be understood as exact 
cancellations of spectral weight owing to parity degeneracy at crossings [see Fig. 1(j)]. This is illustrated in Fig. 2(b) where we plot the transition frequencies weighted by their respective matrix element (represented as the width of the line). Together with the absence of spectral weight of the $\omega_{01}$ transition at crossings, there is a complete spectral weight transfer between higher-energy transitions, where one thick line becomes thin or vice versa (see arrows). Figures 2(c)-2(e) show the $n_{g}$ dependence for three fixed $B$ fields [colored bars in (a)] across a parity crossing. Interestingly, all the spectroscopic features before and after the crossing [(c) and (e), respectively] are shifted exactly by one $e$ unit, which reflects the change of parity of the GS. See also the transitions weighted by their matrix elements in Figs. 2(f)-2(h). This results in distinct spectroscopic features like spectral holes that shift from halfinteger to integer values of $n_{g}$ and vice versa, and changes of curvatures of the involved transitions. Right at the $\delta=0$ parity crossing [Figs. 2(d) and 2(g)], we recover a standard transmon spectrum. Such unique behavior of transmon spectra across a Majorana oscillation should provide a strong signature of the presence of MBSs and their associated parity crossings.

The above behavior has to be contrasted with the spectra resulting from nonoscillating zero modes. Figure 3(a) shows a typical BdG spectrum where a spatially smooth potential $\mu(x)$ in the NW results in a robust zero mode starting at $B \ll B_{c}$. These nontopological zero modes are ubiquitous in experiments and have spurred a longstanding "trivial versus topological" debate in the literature regarding tunneling spectroscopy measurements of zero-bias anomalies [31-35]. Interestingly, the MW response of such robust $B<B_{c}$ zero modes is very distinct from the one we discussed previously for oscillating Majoranas. Here, the overall response of the $B<B_{c}$ zero mode is that of a transmon (but with a main line $\omega_{03}$ corresponding to a parity-conserving transition from an odd GS) and a largely suppressed parity-mixing line $\omega_{01}$ [see Figs. 3(b) and 3(c)]. At a fixed $B<B_{c}$ field (red bar), the $n_{g}$ dependence is that of a transmon with no charge dispersion [see Figs. 3(d) and 3(g)]. In contrast, the appearance of parity crossings which fully develop into oscillating Majoranas at larger $B$ fields [e.g., blue and yellow bars in Fig. 3(b)] results again in an oscillating $\omega_{01}$ line, switches in the higher transitions, and one- $e$ shifts in the gate response [Figs. 3(e) and 3(h) and 3(f) and 3(i)]. Both the amplitude of the oscillations and the intensity of the lowest intraband transition $\omega_{01}$ grow with $B$ field, providing yet another clear signature as compared to the low-frequency response resulting from trivial zero modes at $B<B_{c}$.

In summary, our results demonstrate that MW spectroscopy of NW-based transmon qubits is a powerful tool to detect the presence of oscillating MBSs in the JJ junction. Repeated parity switches in the MW spectrum against magnetic field reveal unambiguously the existence of a subgap state oscillating around zero, which could aid in distinguishing topological Majoranas from other nonoscillating types of zero modes [35], thus providing an alternative to tunneling spectroscopy. Our projection method can be readily extended to other relevant regimes [37]. Other geometries currently under intensive experimental study, including split junctions [37], junctions with quantum dots [51,52], and superconducting
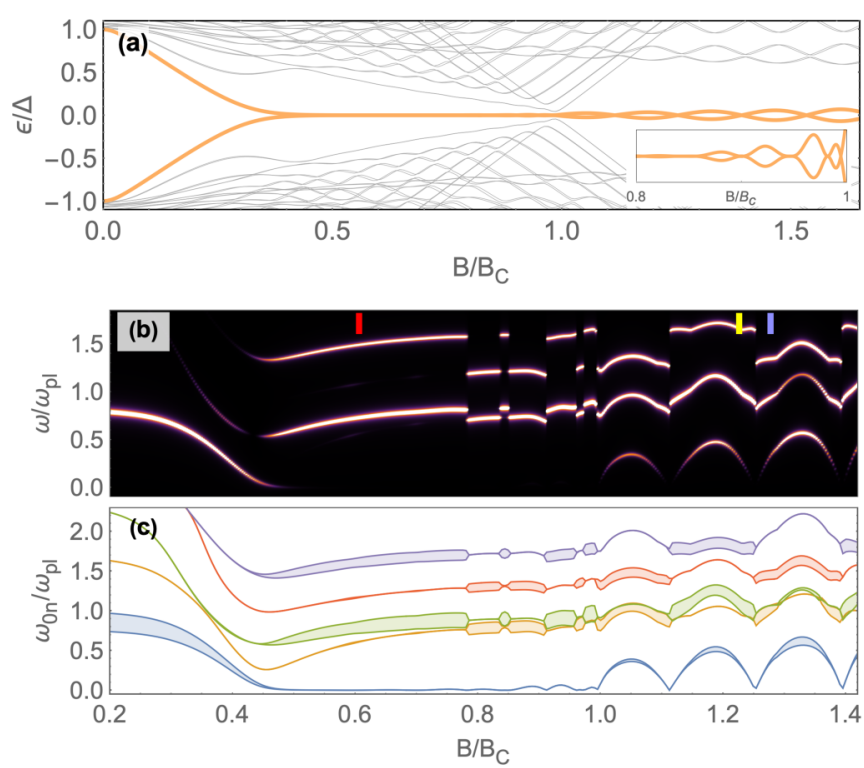
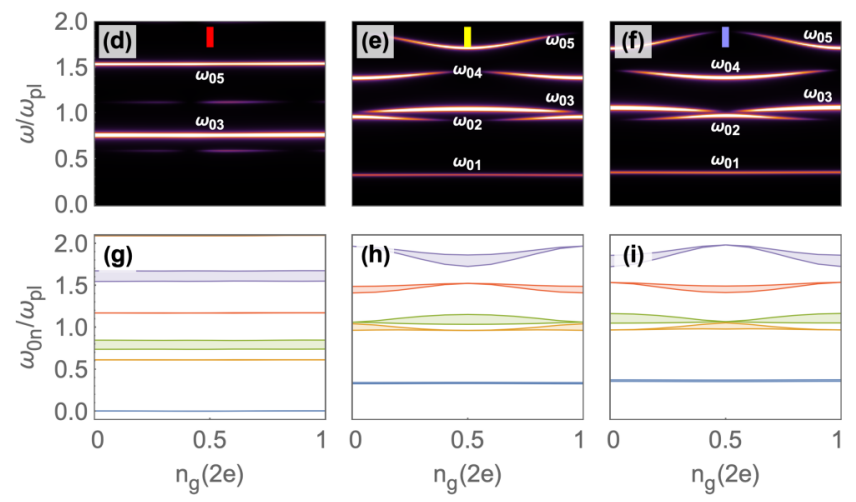

FIG. 3. MW spectroscopy of a junction containing nontopological robust zero modes. (a) The BdG spectrum of a NW with a smooth $\mu(x)$ (which interpolates between $\mu=0.5 \mathrm{meV}$ and $\mu=2 \mathrm{meV}$ at each NW segment) shows robust zero modes for $B<B_{c}$. Inset: blowup near $B \lesssim B_{c}$ where the zero mode starts to develop oscillations. (b), (c) The $B$-field dependence of the MW response (shown here at $n_{g}=0.5$ ) of a transmon containing such wires is distinct depending on whether it is governed by the trivial mode pinned to zero at $B \lesssim 0.8 B_{c}$ or the oscillating Majoranas at $B>B_{c}$. While the former leads to an overall standard transmon response (d), (g), the latter shows parity switches, (e), (h) and (f), (i), as discussed in Fig. 2. Same parameters as Fig. 2.

islands in the fluxonium regime [53], should be the subject of future studies.

\section{ACKNOWLEDGMENTS}

We thank Bernard van Heck for his input. This research is supported by the Spanish Ministry Of Science And Innovation through Grants No. PGC2018-097018-B-I00, No. FIS2016-80434-P (AEI/FEDER, EU), No. BES-2016-078122 (FPI programme), and No. RYC-2011-09345 (Ramón y Cajal programme). The EU Horizon 2020 research and innovation program (FETOPEN Grant Agreement No. 828948) and the CSIC Research Platform on Quantum Technologies PTI-001 are also acknowledged. 
[1] A. Blais, R.-S. Huang, A. Wallraff, S. M. Girvin, and R. J. Schoelkopf, Phys. Rev. A 69, 062320 (2004).

[2] M. H. Devoret and R. J. Schoelkopf, Science 339, 1169 (2013).

[3] G. Wendin, Rep. Prog. Phys. 80, 106001 (2017).

[4] V. Bouchiat, D. Vion, P. Joyez, D. Esteve, and M. H. Devoret, Phys. Scr. T76, 165 (1998).

[5] F. Hassler, A. R. Akhmerov, and C. W. J. Beenakker, New J. Phys. 13, 095004 (2011).

[6] C. Müller, J. Bourassa, and A. Blais, Phys. Rev. B 88, 235401 (2013).

[7] D. Pekker, C.-Y. Hou, V. E. Manucharyan, and E. Demler, Phys. Rev. Lett. 111, 107007 (2013).

[8] P. Virtanen and P. Recher, Phys. Rev. B 88, 144507 (2013).

[9] E. Ginossar and E. Grosfeld, Nat. Commun. 5, 4772 (2014).

[10] K. Yavilberg, E. Ginossar, and E. Grosfeld, Phys. Rev. B 92, 075143 (2015).

[11] O. Dmytruk, M. Trif, and P. Simon, Phys. Rev. B 92, 245432 (2015).

[12] J. I. Väyrynen, G. Rastelli, W. Belzig, and L. I. Glazman, Phys. Rev. B 92, 134508 (2015).

[13] Y. Peng, F. Pientka, E. Berg, Y. Oreg, and F. von Oppen, Phys. Rev. B 94, 085409 (2016).

[14] M. C. Dartiailh, T. Kontos, B. Douçot, and A. Cottet, Phys. Rev. Lett. 118, 126803 (2017).

[15] M. Trif, O. Dmytruk, H. Bouchiat, R. Aguado, and P. Simon, Phys. Rev. B 97, 041415(R) (2018).

[16] A. Keselman, C. Murthy, B. van Heck, and B. Bauer, Sci. Post. Phys. 7, 50 (2019).

[17] T. W. Larsen, K. D. Petersson, F. Kuemmeth, T. S. Jespersen, P. Krogstrup, J. Nygård, and C. M. Marcus, Phys. Rev. Lett. 115, 127001 (2015).

[18] G. de Lange, B. van Heck, A. Bruno, D. J. van Woerkom, A. Geresdi, S. R. Plissard, E. P. A. M. Bakkers, A. R. Akhmerov, and L. DiCarlo, Phys. Rev. Lett. 115, 127002 (2015).

[19] A. Kringhøj, L. Casparis, M. Hell, T. W. Larsen, F. Kuemmeth, M. Leijnse, K. Flensberg, P. Krogstrup, J. Nygård, K. D. Petersson, and C. M. Marcus, Phys. Rev. B 97, 060508(R) (2018).

[20] L. Casparis, T. W. Larsen, M. S. Olsen, F. Kuemmeth, P. Krogstrup, J. Nygård, K. D. Petersson, and C. M. Marcus, Phys. Rev. Lett. 116, 150505 (2016).

[21] F. Luthi, T. Stavenga, O. W. Enzing, A. Bruno, C. Dickel, N. K. Langford, M. A. Rol, T. S. Jespersen, J. Nygård, P. Krogstrup, and L. DiCarlo, Phys. Rev. Lett. 120, 100502 (2018).

[22] L. Casparis, N. J. Pearson, A. Kringhøj, T. W. Larsen, F. Kuemmeth, J. Nygård, P. Krogstrup, K. D. Petersson, and C. M. Marcus, Phys. Rev. B 99, 085434 (2019).

[23] L. Casparis, M. R. Connolly, M. Kjaergaard, N. J. Pearson, A. Kringhøj, T. W. Larsen, F. Kuemmeth, T. Wang, C. Thomas, S. Gronin, G. C. Gardner, M. J. Manfra, C. M. Marcus, and K. D. Petersson, Nat. Nanotechnol. 13, 915 (2018).

[24] J. G. Kroll, W. Uilhoorn, K. L. van der Enden, D. de Jong, K. Watanabe, T. Taniguchi, S. Goswami, M. C. Cassidy, and L. P. Kouwenhoven, Nat. Commun. 9, 4615 (2018).

[25] F. E. Schmidt, M. D. Jenkins, K. Watanabe, T. Taniguchi, and G. A. Steele, Nat. Commun. 9, 4069 (2018).

[26] J. I.-J. Wang, D. Rodan-Legrain, L. Bretheau, D. L. Campbell, B. Kannan, D. Kim, M. Kjaergaard, P. Krantz, G. O. Samach,
F. Yan, J. L. Yoder, K. Watanabe, T. Taniguchi, T. P. Orlando, S. Gustavsson, P. Jarillo-Herrero, and W. D. Oliver, Nat. Nanotechnol. 14, 120 (2019).

[27] R. M. Lutchyn, J. D. Sau, and S. Das Sarma, Phys. Rev. Lett. 105, 077001 (2010).

[28] Y. Oreg, G. Refael, and F. von Oppen, Phys. Rev. Lett. 105, 177002 (2010).

[29] R. Aguado, Riv. Nuovo Cimento 40, 523 (2017).

[30] R. M. Lutchyn, E. P. A. M. Bakkers, L. P. Kouwenhoven, P. Krogstrup, C. M. Marcus, and Y. Oreg, Nat. Rev. Mater. 3, 52 (2018).

[31] C. Moore, T. D. Stanescu, and S. Tewari, Phys. Rev. B 97, 165302 (2018).

[32] F. Peñaranda, R. Aguado, P. San-Jose, and E. Prada, Phys. Rev. B 98, 235406 (2018).

[33] A. Vuik, B. Nijholt, A. R. Akhmerov, and M. Wimmer, Sci. Post. Phys. 7, 61 (2019).

[34] J. Avila, F. Peñaranda, E. Prada, P. San-Jose, and R. Aguado, Commun. Phys. 2, 133 (2019).

[35] E. Prada, P. San-Jose, M. W. A. de Moor, A. Geresdi, E. J. H. Lee, J. Klinovaja, D. Loss, J. Nygård, R. Aguado, and L. P. Kouwenhoven [Nat. Rev. Phys. (to be published)], arXiv: 1911.04512.

[36] J. Cayao, P. San-Jose, A. M. Black-Schaffer, R. Aguado, and E. Prada, Phys. Rev. B 96, 205425 (2017).

[37] J. Ávila, E. Prada, P. San-Jose, and R. Aguado, Phys. Rev. B 102, 094518 (2020).

[38] P. San-Jose, E. Prada, and R. Aguado, Phys. Rev. Lett. 108, 257001 (2012).

[39] D. I. Pikulin and Y. V. Nazarov, Phys. Rev. B 86, 140504(R) (2012).

[40] The possibility of trivial Andreev levels has also been analyzed within the framework of the effective low-energy model in Ref. [54]. Note, however, that the scenario discussed in that reference, a quantum dot in a topological insulator wire, cannot lead to the robust zero modes discussed here but only to accidental zero-energy crossings of Andreev levels.

[41] P. San-Jose, J. Cayao, E. Prada, and R. Aguado, New J. Phys. 15, 075019 (2013).

[42] P. San-Jose, E. Prada, and R. Aguado, Phys. Rev. Lett. 112, 137001 (2014).

[43] S. M. Albrecht, A. P. Higginbotham, M. Madsen, F. Kuemmeth, T. S. Jespersen, J. Nygård, P. Krogstrup, and C. M. Marcus, Nature (London) 531, 206 (2016).

[44] J. Shen, S. Heedt, F. Borsoi, B. van Heck, S. Gazibegovic, R. L. M. Op het Veld, D. Car, J. A. Logan, M. Pendharkar, S. J. J. Ramakers, G. Wang, D. Xu, D. Bouman, A. Geresdi, C. J. Palmstrøm, E. P. A. M. Bakkers, and L. P. Kouwenhoven, Nat. Commun. 9, 4801 (2018).

[45] R. V. Mishmash, D. Aasen, A. P. Higginbotham, and J. Alicea, Phys. Rev. B 93, 245404 (2016).

[46] This claim is only true for spatially homogeneous wires since a smooth $\mu(x)$ can lead to $\delta \rightarrow 0$ irrespective of the ratio $\xi_{M} / L_{S}$, as we discuss in Fig. 3.

[47] J. Tiira, E. Strambini, M. Amado, S. Roddaro, P. San-Jose, R. Aguado, F. S. Bergeret, D. Ercolani, L. Sorba, and F. Giazotto, Nat. Commun. 8, 14984 (2017).

[48] Considering typical critical current values $I_{c} \sim 0.2 I_{0}$, with $I_{0}=$ $e \Delta / \hbar$ the maximum supercurrent of a single open channel, this 
gives $E_{J} \sim 0.1 \Delta \sim 25 \mu \mathrm{eV} \sim 6 \mathrm{GHz}$, assuming an induced gap of the order of $\Delta \sim 250 \mu \mathrm{eV}$. Since $E_{M} \sim E_{J}$ for a topological single channel, and considering typical charging energies for transmons $E_{C} \sim 200-300 \mathrm{MHz}$ [17-19], the junction is naturally in the $E_{M} / E_{C} \gg 1$ regime.

[49] M. F. Goffman, C. Urbina, H. Pothier, J. Nygard, C. M. Marcus, and P. Krogstrup, New J. Phys. 19, 092002 (2017).

[50] L. Tosi, C. Metzger, M. F. Goffman, C. Urbina, H. Pothier, S. Park, A. L. Yeyati, J. Nygård, and P. Krogstrup, Phys. Rev. X 9 , 011010 (2019).
[51] A. Bargerbos, W. Uilhoorn, C.-K. Yang, P. Krogstrup, L. P. Kouwenhoven, G. de Lange, B. van Heck, and A. Kou, Phys. Rev. Lett. 124, 246802 (2020).

[52] A. Kringhøj, B. van Heck, T. W. Larsen, O. Erlandsson, D. Sabonis, P. Krogstrup, L. Casparis, K. D. Petersson, and C. M. Marcus, Phys. Rev. Lett. 124, 246803 (2020).

[53] M. Pita-Vidal, A. Bargerbos, C.-K. Yang, D. J. van Woerkom, W. Pfaff, N. Haider, P. Krogstrup, L. P. Kouwenhoven, G. de Lange, and A. Kou, arXiv:1910.07978.

[54] K. Yavilberg, E. Ginossar, and E. Grosfeld, Phys. Rev. B 100, 241408(R) (2019). 